\title{
Smoking among hospitalized patients: A multi-hospital cross-sectional study of a widely neglected problem
}

\author{
Cristina Martínez',2, Marcela Fu', ${ }^{1,3}$ Yolanda Castellano', Anna Riccobene', Paz Fernández2,4, Sandra Cabrera4, Eva Gavilan ${ }^{1,5}$, \\ Ariadna Feliu',3, Montse Puig-Llobet², Pilar Fuster ${ }^{5}$, Jose María Martínez-Sánchez ${ }^{5}$, Javier Montes ${ }^{6}$, Joan Maria Estrada², \\ Carmen Moreno", Anna Falcó-Pegueroles², Jordi Galimany², Cecilia Brando ${ }^{6}$, Rosa Suñer-Soler7, Anna Capsada ${ }^{8}$, Esteve \\ Fernández ${ }^{1,3}$, y Grupo de Coordinadores de la Red Catalana de Hospitales sin Humo (XCHsF)
}

\section{ABSTRACT}

INTRODUCTION A comprehensive smoking ban was recently enacted for acute-care hospital campuses in Spain. The aim of this study was to assess the prevalence and patterns of smoking among inpatients before and during hospitalization. METHODS Multi-center cross-sectional study was conducted in 13 hospitals in the province of Barcelona, Spain from May 2014 to May 2015. Participants were adults who provided informed consent. The sample size was calculated to be representative of each hospital (prevalence $29.4 \%$, precision $\pm 5 \%$, error $5 \%)$. We approached 1228 subjects, 888 accepted to participate and 170 were replaced (were not available or declined to participate). Final sample comprised 1047 subjects. We used a computer-assisted personal interview system to collect data, including sociodemographic variables and use of tobacco before and during hospitalization. Smoking status was validated with exhaled carbon monoxide. We calculated overall tobacco prevalence and investigated associations with participant and center characteristics. We performed multiple polytomous and multilevel logistic regression analyses to estimate odds ratios (ORs) and 95\% confidence intervals (CIs), with adjustments for potential confounders.

RESULTS In all, 20.5\% (95\% CI: 18.1-23.0) of hospitalized patients were smokers. Smoking was most common among men ( $\mathrm{aOR}=7.47$; 95\% CI: 4.88-11.43), young age groups (18-64 years), and individuals with primary or less than primary education $(\mathrm{aOR}=2.76 ; 95 \% \mathrm{CI}$ : $1.44-5.28)$. Of the smokers, $97.2 \%$ were daily consumers of whom $44.9 \%$ had medium nicotine dependence. Of all smokers, three-quarters expressed a wish to quit, and one-quarter admitted to consuming tobacco during hospitalization.

CONCLUSIONS Our findings indicate the need to offer smoking cessation interventions among hospitalized patients in all units and service areas, to avoid infringements and increase patient safety, hospital efficiency, and improve clinical outcomes. Hospitalization represents a promising window for initiating smoking interventions addressed to all patients admitted to smoke-free hospitals, specially after applying a smoke-free campus ban.

\section{AFFILIATION}

1 Tobacco Control Unit, Cancer Control and Prevention Programme, Institut Català d'Oncologia-ICOIDIBELL, Barcelona, Spain 2 School of Nursing, Faculty of Medicine and Health Sciences, University of Barcelona, Barcelona, Spain

3 School of Medicine, Department of Clinical Sciences, Universitat de Barcelona, Barcelona, Spain 4 Nursing Research Unit, Institut Català d'Oncologia-ICO, Barcelona, Spain

5 Medicine and Health Sciences School, Universitat Internacional de Catalunya, Barcelona, Spain 6 Department of Nursing Science, Gimbernat School, Barcelona, Spain

7 Department of Nursing Science, University of Girona, Girona, Spain 8 Fundació Althaia, Barcelona, Spain

CORRESPONDENCE TO

Cristina Martínez. Tobacco Control Unit, Institut Català d'Oncologia, Av. Gran Via de L'Hospitalet, 199-203, E-08908 L'Hospitalet de Llobregat, Barcelona, Spain.

E-mail: cmartinez@iconcologia.net

KEYWORDS

smoking, tobacco, epidemiology, policy, health services

Received: 11 April 2018

Revised: 25 June 2018

Accepted: 5 July 2018

\section{INTRODUCTION}

Tobacco consumption is responsible for one-sixth of the 6 million annual deaths caused by non- communicable diseases worldwide, including cardiovascular diseases, chronic respiratory diseases and cancer ${ }^{1}$. In 2005, the Framework Convention on 
Tobacco Control (FCTC) promoted several policies to tackle the tobacco epidemic. Of these policies, Article 8 proposed smoking bans and Article 14 directed countries to implement effective programs to assist individuals in quitting tobacco $\mathrm{use}^{2}$. In addition, the FCTC asserted that health organizations and healthcare professionals should act as examples in controlling tobacco consumption, championing compliance with the law, and providing smoking cessation aids ${ }^{3}$.

The scientific literature has shown that tobacco control policies adopted in healthcare organizations have mainly achieved changes in organizations and workers. These changes include reductions in smoking prevalence, increases in the number of attempts to quit smoking among health professionals $^{4-6}$, and increases in the number of tobacco cessation interventions available $\mathrm{e}^{7,8}$. However, only a small number of these studies have evaluated the impact of these measures on patients that consume tobacco. Smoking among hospitalized patients continues to be a widely neglected problem. Hospitalized patients exhibit a high smoking prevalence and frequent infringements of smokefree policies ${ }^{9-11}$. In Spain, the tobacco epidemic is at Stage IV, according to the cigarette epidemic model. This model shows a marked downturn in smoking prevalence among men and women and a decline of deaths attributable to smoking among men, but an increase among women ${ }^{12}$. According to the latest Spanish National Health Survey, tobacco consumption among adults ( $\geqslant 15$ years old) has dropped from $38.7 \%$ in $2001^{13}$ to $25.4 \%$ in $2014^{14}$. Despite this important decline, tobaccorelated diseases continue to cause $15.2 \%$ of the total mortality in Spain ${ }^{15}$. Approximately 5 million individuals receive medical or surgical treatment in acute-care hospitals annually in Spain ${ }^{16}$, of whom about 1 million are smokers.

In 2011, Spain established one of the most comprehensive tobacco control regulation policies in Europe for healthcare services ${ }^{17}$. The Spanish legislation pioneered an innovative legal framework that banned smoking in indoor and outdoor areas of acute-care hospitals. Responsibility was given to hospital management and health regional administrators to provide effective smoking cessation interventions, including counseling and pharmacological therapy, to address the needs of hospitalized patients that consumed tobacco ${ }^{18}$.

Once the new Spanish legislation was established, the opportunity arose for evaluating the impact of tobacco control laws among inpatients and for assessing determinants of smoking during hospitalization. The ultimate goal of these studies is to improve our understanding of the needs of patients that attempt to quit smoking. This information would facilitate the design of tobacco cessation services and interventions for initiating attempts to quit smoking in a supportive, smokefree environment. Therefore, this study aimed to assess the prevalence, determinants, and patterns of smoking before and during hospital admission, in 13 hospitals in the province of Barcelona, Spain.

\section{METHODS}

\section{Design}

In this multi-center cross-sectional study, we conducted a survey of a random sample of hospitalized patients admitted to 13 acute-care hospitals in Barcelona province (Northeast Spain), which comprises an area of $7.733 \mathrm{~km}^{2}$ of 5.5 million inhabitants. Hospitals were selected by convenience from the 38 acute-care hospitals that belonged to the Catalan Network for Smoke-free Hospitals (XCHsF, www.xchsf.com) in Barcelona province.

\section{Participants}

Volunteers participated in the study. Participants were hospitalized adults ( $\geqslant 18$ years), conscious and oriented in space, time, and person, with a stay $\geqslant 24$ hours. All participants provided informed consent. Patients that were hospitalized from the emergency room and intensive care units were excluded.

Sample sizes were calculated to be representative of each hospital and, after weighting, representative of all of Catalonia. Estimation of the sample size for each hospital took into account the total number of acute-care beds and the available regional smoking prevalence in Catalonia $(29.4 \%)^{19}$. The required sample size was 1034 subjects, assuming a precision of $\pm 5 \%$ and an error of $5 \%$. The calculation was performed with Statcalc in EpiInfo, version 6.0.4 (Centers for Disease Control and Prevention, Atlanta, US).

In each hospital, individuals were randomly selected from the daily updated admission list. The 
randomization system for selecting participants was based on four steps: 1) the number of cases in each hospital was divided by the number of beds, 2) a random number was selected between one and the value attained in step one, 3 ) the first selection was the case number that matched the number chosen in step two, 4) the next case was selected by adding the selected number in step two to the number obtained in step one. For example, for a hospital with 350 beds, the sample size needed to account for 70 patients. Next, we divided the bed number (350) of the hospital by the sample number (70) of participants selected, i.e. $350 / 70=5$. We chose a random number between 1 and 5 (e.g. 3). We began selecting the participants with that number (e.g. 3) and the 5 th case patients were invited to participate. Thus, our invitation list became: $3,8,13,18$, and so on.

When a selected patient corresponded to an empty bed or a patient that was unavailable (for instance, in a test) or when the patient declined to participate, we invited the next patient on the list that fulfilled the inclusion and exclusion criteria. In each survey, this type of substitution accounted for less than $16 \%$ of the corresponding sample. Thus, we approached 1228 subjects in total, 888 accepted to participate and 170 were replaced $(80 \%$ were not available and $20 \%$ declined to participate at the time of the interview). From the overall 1058 questionnaires obtained, 9 were excluded from the analysis because $>20 \%$ of their content was blank (Figure 1).

\section{Data collection}

We used an ad hoc questionnaire developed by an expert working group from the XCHsF (www. xchsf.com). This questionnaire was composed of 86 questions exploring several dimensions, however for the purpose of this study the main outcome variable was tobacco consumption that was defined according to WHO criteria $^{20}$. Respondents were classified

Figure 1. Flowchart of the recruitment process

Sample size criteria

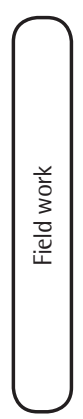

Hospitals: 13

Acute Beds: 5526

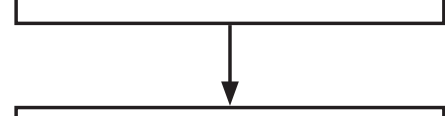

Expected prevalence: $29.4 \%$

Precision: + /-5\% Error 5\%

Required sample size $=1034$ subjects

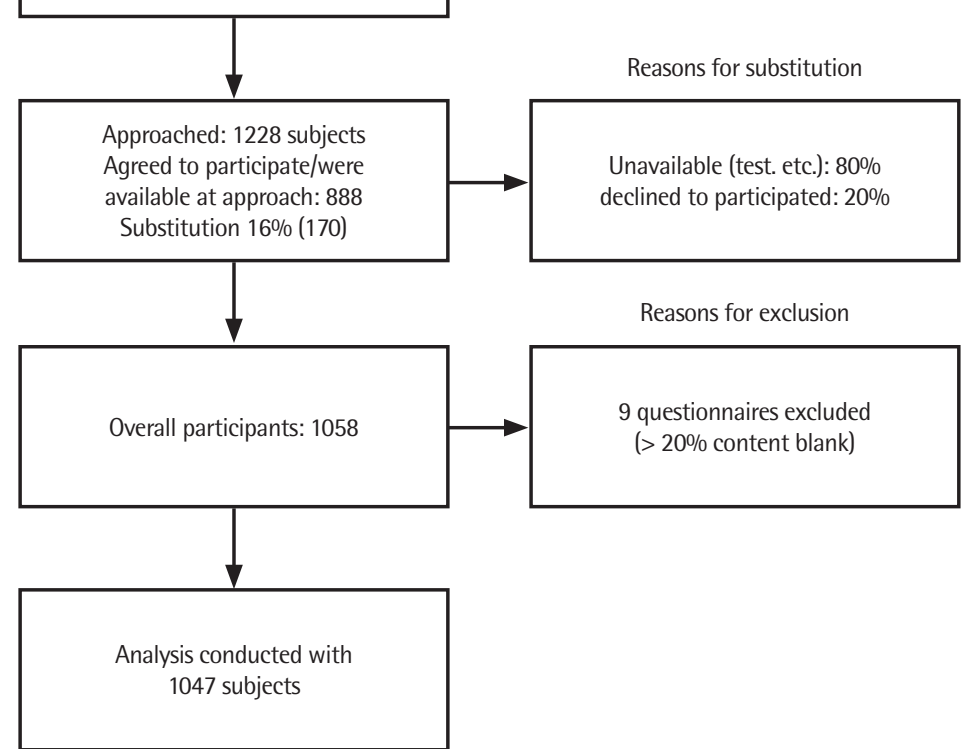


into three categories: 1) current smoker, defined as a person that smoked regularly, either daily (at least one cigarette per day - CPD) or occasionally (less than one CPD) at the time of the survey; 2) non-smoker, defined as a person that had never smoked or had smoked less than 100 cigarettes in his/her lifetime; and 3) former smoker, defined as a person that smoked in the past, but had quit at least 6 months prior to the study. Subjects were asked about their tobacco consumption before and during their hospital admission.

To assess smoking patterns, current and former smokers were asked at what age they had started smoking and the number of cigarettes smoked per day. Among current smokers, we assessed what type of tobacco product they used (cigarettes, roll your own - RYO, electronic cigarettes, cigars, or pipe); the number of cigarettes smoked per day (CPD; classified as $<10,10-19$, and $\geqslant 20$ ); and the time to the first cigarette after waking ( $\leqslant 5,6-30,31-60,>60 \mathrm{~min})$. Nicotine dependence was assessed with the Heavy Smoking Index (HSI), a six-point scale calculated from the number of cigarettes smoked per day and the time to the first cigarette after waking. The HSI scores were categorized into three levels of nicotine dependence: low (0-2), medium (3-4), and high (5$6)^{21}$. Withdrawal symptoms were assessed according to DSM-IV categories. Smokers also reported whether they had abstained from smoking during hospitalization (yes/no). We measured exhaled carbon monoxide $(\mathrm{CO})$ to validate abstinence with a CO-oximeter. The cutoff value for active tobacco consumption was set at 6 particles per million $(\mathrm{ppm})^{22}$. We assessed the readiness to quit with the Prochaska and DiClemente change model stages that include: pre-contemplative, contemplative, active, maintenance, and relapse ${ }^{23}$.

The main descriptive variables relating to patients were: 1) sociodemographics, such as sex and age (classified as $<45$ years, $45-64$ years, $>64$ years, based on the median distribution); 2) educational level (less than primary, primary, high school, and university); 3 ) occupation (employed, unemployed, retired, other - students and homecare roles); 4) partner's smoking status (no partner, non-smoking partner, smoking partner); 5) self-perceived health status (excellent, very good, good, adequate, or poor); 6) independence index, according to the
Barthel index (100 independent, $<100$ dependent); and 7) comorbidities.

In addition, we explored the following variables relating to hospitals: 1) the level of technology of the center (general or high-technology hospital); 2) type of ward (the original ward was noted, but later categorized as surgical, medical-surgical, or medical); $3)$ number of beds $(\leqslant 300,>300)$; 4) smoking prevalence among health professionals $(\leqslant 30 \%,>30 \%$, based on records in the Catalan Network for Smokefree Hospitals files (XCHsF); 5) smoking cessation program at the hospital (yes, no); 6) accreditation level, according to XCHsF standards (gold, silver, bronze, and member).

The questionnaire had been tested previously with a pilot sample of participants in one hospital. During the pilot testing a couple of questions were slightly changed mainly because interviewers mentioned that patients did not understand them. For example, instead of employing the term 'e-health' we asked current smokers and former smokers whether they have used webs, apps, and other Internet resources for quitting. We collected data with a computerassisted personal interview (CAPI) system. Trained interviewers conducted the interviews face-to-face, from May 2014 to May 2015.

\section{Ethical considerations}

The study protocol was approved by the Ethics Committee of the Hospital Universitari de Bellvitge (PR234/11). All participants provided written consent to participate.

\section{Data analysis}

Descriptive analyses were performed on participant and center characteristics. Tobacco consumption prevalence was computed overall, according to sex, and for each center. Direct standardization was used to control for age. The reference population was all the participants in the study, and the following age groups were used for standardization: 18-29, 30-39, $40-49,50-59,60-69$, and $\geqslant 70$ years.

The main outcome variable was smoking status (current smoker, former smoker, and non smoker). A bivariate analysis was carried out with logistic regression to estimate the association between the main outcome variables and the sociodemographic and center characteristics. To identify the main 
determinants that characterized a smoker and/ or former smoker, we fitted polytomous logistic regression models. Results are presented as crude odds ratio (cOR) or adjusted OR (aOR), as a measure of association. We used a multilevel logistic regression model, due to the variability in the prevalence of tobacco use among centers. Statistical significance was set at $\mathrm{p}<0.05$. Analyses were conducted with SPSS version 21 and STATA version 13. The weights from the sample design were applied to all calculations.

\section{RESULTS}

\section{Sociodemographic data}

The final sample comprised 1047 subjects. The number of participants per hospital ranged from 41 to 205. Table 1 shows the participant

\section{Table 1. Smoking status according to variables that represent patient characteristics (Hospitals of Barcelona} Province, 2014-2015)

\begin{tabular}{|c|c|c|c|c|c|c|c|c|c|c|c|c|}
\hline & \multicolumn{3}{|c|}{ Overall } & \multicolumn{3}{|c|}{ Current smokers } & \multicolumn{3}{|c|}{ Former smokers } & \multicolumn{3}{|c|}{ Von-smokers } \\
\hline & n & $\% 1$ & $95^{\circ} \circ \mathrm{CI}$ & n & $0^{2}$ & $95^{\circ} \circ \mathrm{CI}$ & n & $0^{2}$ & $95 \% \mathrm{CI}$ & n & $\%^{2}$ & $95^{\circ} \circ \mathrm{CI}$ \\
\hline Overall & 1047 & 100 & - & 215 & 20.5 & $(18.1-23.0)$ & 346 & 33.1 & $(30.2-35.9)$ & 486 & 46.4 & $(43.4-49.4)$ \\
\hline \multicolumn{13}{|l|}{ Sex } \\
\hline Female & 520 & 49.7 & $(46.6-52.7)$ & 70 & 13.5 & $(10.5-16.4)$ & 95 & 18.3 & $(14.9-21.6)$ & 355 & 68.2 & $(64.3-72.3)$ \\
\hline Male & 527 & 50.3 & $(47.3-53.4)$ & 145 & 27.5 & $(23.7-31.3)$ & 251 & 47.6 & $(43.4-51.9)$ & 131 & 24.9 & $(21.2-28.5)$ \\
\hline \multicolumn{13}{|l|}{ Age groups (years) } \\
\hline$<45$ & 255 & 24.4 & $(21.8-27.0)$ & 81 & 31.8 & $(26.1-37.5)$ & 49 & 19.2 & $(14.4-24.1)$ & 125 & 49.0 & $(42.9-55.2)$ \\
\hline $45-64$ & 314 & 30.0 & $(27.2-32.8)$ & 96 & 30.6 & $(25.5-35.7)$ & 122 & 38.8 & $(33.5-44.2)$ & 96 & 30.6 & $(25.5-35.7)$ \\
\hline$>64$ & 478 & 45.6 & $(42.6-48.7)$ & 38 & 7.9 & $(5.5-10.4)$ & 175 & 36.7 & $(32.3-40.9)$ & 265 & 55.4 & $(51.0-59.9)$ \\
\hline \multicolumn{13}{|l|}{ Education } \\
\hline Less than prir & 360 & 34.5 & $(31.6-37.4)$ & 46 & 12.8 & $(9.3-16.2)$ & 113 & 31.4 & $(26.6-36.2)$ & 201 & 55.8 & $(50.7-61.0)$ \\
\hline Primary & 330 & 31.6 & $(28.8-34.4)$ & 95 & 28.8 & $(23.9-33.7)$ & 111 & 33.6 & $(28.5-38.7)$ & 124 & 37.6 & $(32.4-42.8)$ \\
\hline High School & 213 & 20.4 & $(18.0-22.8)$ & 55 & 25.8 & $(19.9-31.7)$ & 68 & 31.9 & $(25.7-38.2)$ & 90 & 42.3 & $(35.6-48.9)$ \\
\hline University & 141 & 13.5 & $(11.4-15.6)$ & 19 & 13.5 & $(7.8-19.1)$ & 54 & 38.3 & $(30.3-46.3)$ & 68 & 48.2 & $(40.0-56.5)$ \\
\hline \multicolumn{13}{|l|}{ Occupation } \\
\hline Employed & 321 & 30.7 & $(27.9-33.5)$ & 101 & 31.5 & $(26.4-36.5)$ & 92 & 28.7 & $(23.7-33.6)$ & 128 & 39.8 & $(34.5-45.2)$ \\
\hline Unemployed & 77 & 7.4 & $(5.8-8.9)$ & 30 & 38.9 & $(28.1-49.9)$ & 17 & 22.1 & $(12.8-31.3)$ & 30 & 39.0 & $(28.1-49.9)$ \\
\hline Retired & 532 & 50.7 & $(47.8-53.8)$ & 75 & 14.1 & $(11.1-17.1)$ & 227 & 42.7 & $(38.5-46.9)$ & 230 & 43.2 & $(39.0-47.4)$ \\
\hline Others & 117 & 11.2 & $(9.3-13.1)$ & 9 & 7.7 & $(2.9-12.5)$ & 10 & 8.5 & $(3.5-13.6)$ & 98 & 83.8 & $(77.1-90.4)$ \\
\hline \multicolumn{13}{|l|}{ Partner's smoking status } \\
\hline No partner & 312 & 29.9 & $(27.1-32.6)$ & 74 & 23.7 & $(19.0-28.4)$ & 76 & 24.4 & $(19$ & 162 & 51.9 & $-57.5)$ \\
\hline Non-smoker partner & 563 & 53.8 & $(50.9-56.9)$ & 68 & 12.1 & $(9.4-14.8)$ & 224 & 39.8 & $(35.7-43.8)$ & 271 & 48.1 & $(44.0-52.3)$ \\
\hline Smoker partner & 170 & 16.3 & $(14.0-18.5)$ & 72 & 42.3 & $(34.9-49.8)$ & 46 & 27.1 & $(20.4-33.7)$ & 52 & 30.6 & $(23.7-37.5)$ \\
\hline \multicolumn{13}{|l|}{ Perceived health status } \\
\hline Excellent/Very good & 64 & 15.7 & $(13.5-17.9)$ & 39 & 23.8 & $(17.3-3$ & 39 & 23.8 & $(17.3-3$ & 86 & 52.4 & $(44$ \\
\hline Good & 3 & 43.2 & $(40.3-46.3)$ & 100 & 22.1 & $(18.3-$ & 148 & 32.7 & & 205 & 45.2 & \\
\hline Adequate/Poor & 0 & 41.1 & $(38.1-44.0)$ & 76 & 17.7 & $(14.1-21.3)$ & 159 & 37.0 & $(32.4-41.5)$ & 195 & 45.3 & $(40.6-50.1)$ \\
\hline \multicolumn{13}{|l|}{ Barthel Index } \\
\hline Dependent $(<100)$ & 230 & 22.0 & $(19.5-24.5)$ & 28 & 12.2 & $(7.9-16.4)$ & 83 & 36.1 & $(29.9-42.3)$ & 119 & 51.7 & $(45.3-58.2)$ \\
\hline Independent (100) & 817 & 78.0 & $(75.5-80.5)$ & 187 & 22.9 & $(20.0-25.8)$ & 263 & 32.2 & $(29.0-35.4)$ & 367 & 44.9 & $(41.5-48.3)$ \\
\hline \multicolumn{13}{|l|}{ Comorbidities* } \\
\hline Arterial hypertension & 431 & 41.2 & $(38.2-44.1)$ & 56 & 13.0 & $(9.8-16.2)$ & 166 & 38.5 & $(33.9-4$ & 209 & 48.5 & $(43$ \\
\hline Diabetes & 270 & 25.8 & $(23.1-28.4)$ & 33 & 12.2 & $(8.3-16.1)$ & 106 & 39.3 & $(33.4-45.1)$ & 131 & 48.5 & $(42.6-54.5)$ \\
\hline Pneumonia & 216 & 20.6 & $(18.2-23.1)$ & 30 & 13.9 & $(9.3-18.5)$ & 103 & 47.7 & $(41.0-54.3)$ & 83 & 38.4 & $(31.9-44.9)$ \\
\hline Kidney diseases & 201 & 19.2 & $(16.8-21.6)$ & 24 & 11.9 & $(7.5-16.4)$ & 93 & 46.3 & $(39.4-53.2)$ & 84 & 41.8 & $(35.0-48.6)$ \\
\hline Chronic liver diseases & 129 & 12.3 & $(10.3-14.3)$ & 30 & 23.3 & $(16.0-30.5)$ & 49 & 38.0 & $(29.6-46.4)$ & 50 & 38.7 & $(30.4-47.2)$ \\
\hline Cancer & 256 & 24.5 & $(21.8-27.1)$ & 45 & 17.6 & $(12.9-22.2)$ & 105 & 41.0 & $(35.0-47.0)$ & 106 & 41.4 & $(35.4-47.4)$ \\
\hline Heart diseases & 258 & 24.6 & $(22-27.3)$ & 33 & 12.8 & $(8.7-16.9)$ & 99 & 38.4 & $(32.4-44.3)$ & 126 & 48.8 & $(42.7-54.9)$ \\
\hline Cerebrovascular diseases & 108 & 10.3 & $(8.5-12.2)$ & 13 & 12.0 & $(5.9-18.2)$ & 35 & 32.4 & $(23.6-41.2)$ & 60 & 55.6 & $(46.2-64.9)$ \\
\hline Respiratory diseases & 208 & 19.9 & $(17.4-22.3)$ & 44 & 21.2 & $(15.6-26.7)$ & 84 & 40.3 & $(33.7-47.1)$ & 80 & 38.5 & $(31.8-45.1)$ \\
\hline \multicolumn{13}{|l|}{ Exhaled carbon monoxide } \\
\hline $\mathrm{CO} \leq 6 \mathrm{ppm}$ & 588 & 56.2 & $(53.2-59.2)$ & 78 & 13.3 & $(10.5-16.0)$ & 197 & 33.5 & $(29.7-37.3)$ & 313 & 53.2 & $(49.2-57.3)$ \\
\hline $\mathrm{CO}>6 \mathrm{ppm}$ & 260 & 24.8 & $(22.2-27.4)$ & 103 & 39.6 & $(33.7-45.6)$ & 78 & 30.0 & $(24.4-35.6)$ & 79 & 30.4 & $(24.8-36.0)$ \\
\hline
\end{tabular}

${ }^{*}$ Multiple responses allowed. Cl: confidence interval. 1 Column \%. 2 Row \%. 
sociodemographic characteristics and the main variables. In brief, half of participants were men, predominantly $>64$ years old $(45.6 \%)$. The majority had less than primary or primary education $(66.1 \%)$, were retired $(50.6 \%)$, and with a nonsmoking partner (53.8\%). Before hospitalization, most declared that they were in good $(43.2 \%)$ or adequate to poor health (41.1\%), and most $(78.0 \%)$ completed daily activities independently (without assistance).

Overall, 20.5\% (95\% CI: 18.1-23.0) of respondents were current smokers; this proportion varied by hospital, with a range of 14.7 to $30.3 \%$ (Figure 2). By sex, $27.5 \%$ of men were smokers (95\% CI: $23.7-$ 31.3 ) and $13.5 \%$ were women (95\% CI: 10.5-16.4). The highest smoking prevalence was observed in the youngest group, $<45$ years, for both men and women (men 47.2\%, 95\% CI: 36.8-57.6; women 23.5\%, 95\% CI: 17.0-29.9), compared to the middle age group, 45-64 years, (men 38.1\%, 95\% CI: 31.2-45.0; women $19.2 \%, 95 \%$ CI: $12.3-26.1$ ), and the older age group, $>64$ years, (men 12.4\%, 95\% CI: 8.3-16.6; women $3.1 \%$, 95\% CI: 0.8-5.3). Among the current smokers, $38.9 \%$ were unemployed, $31.5 \%$ were employed, and $42.3 \%$ had a partner that also smoked.

Former smokers comprised $33.1 \%$ of all patients, and significantly different proportions of former smokers were observed between the sexes and among different age groups (Table 1).

\section{Smoking status according to hospital characteristics}

Table 2 summarizes the smoking status of patients in hospitals with different characteristics. The lowest
Figure 2. Age-standardized prevalence (\%) of smoking, in participant hospitals of Barcelona Province,

2014-2015 (The area of each dot represents the relative weighting of the hospital, in terms of the proportion of participants among the total, bars represent $95 \%$ CI, the diamond and the dotted line represent the overall weighted prevalence and $95 \% \mathrm{CI}$ )

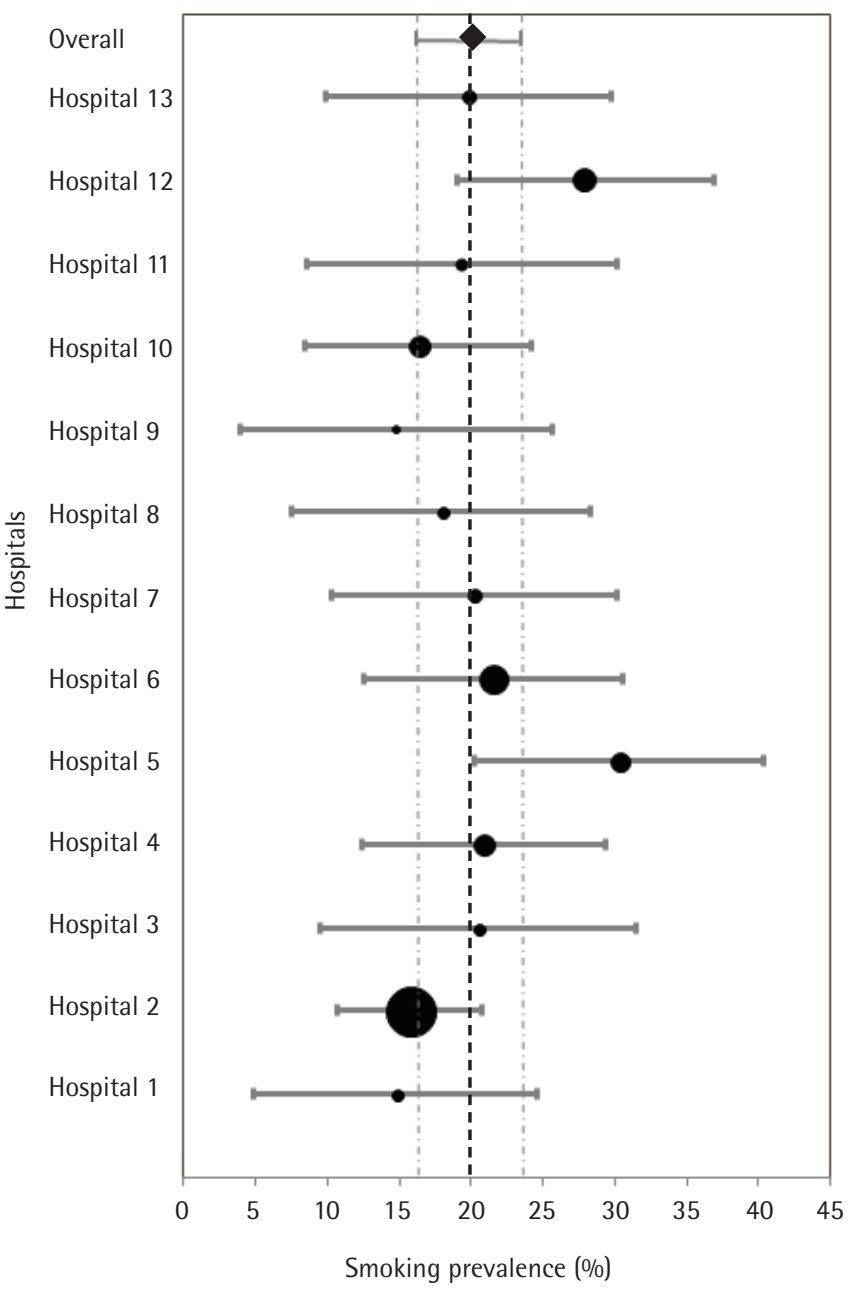

Table 2. Smoking status according to variables that represent hospital characteristics (Hospitals of Barcelona Province, 2014-2015)

\begin{tabular}{|c|c|c|c|c|c|c|c|c|c|c|c|c|}
\hline & \multicolumn{3}{|c|}{ Overall } & \multicolumn{3}{|c|}{ Current smokers } & \multicolumn{3}{|c|}{ Former smokers } & \multicolumn{3}{|c|}{ Von-smokers } \\
\hline & n & ${ }_{0}^{1}$ & $95^{\circ} \circ \mathrm{CI}$ & 11 & $0^{2}$ & $95^{\circ} \circ \mathrm{CI}$ & n & $0^{2}$ & $95^{\circ} \circ \mathrm{CI}$ & n & $00^{2}$ & $95^{\circ} \circ \mathrm{OI}$ \\
\hline \multicolumn{13}{|l|}{ Level of center } \\
\hline General hospital & 245 & 23.4 & $(20.8-26.0)$ & 54 & 22.0 & $(16.9-27.2)$ & 80 & 32.7 & $(26.8-38.5)$ & 111 & 45.3 & $(39.1-51.5)$ \\
\hline High-technology hospital & 802 & 76.6 & $(74.0-79.2)$ & 161 & 20.1 & $(17.3-22.8)$ & 266 & 33.2 & $(29.9-36.4)$ & 375 & 46.7 & $(43.3-50.2)$ \\
\hline \multicolumn{13}{|l|}{ Type of ward } \\
\hline Surgical & 361 & 34.5 & $(31.6-37.4)$ & 75 & 20.8 & $(16.6-25.0)$ & 118 & 32.7 & $(27.8-37.5)$ & 168 & 46.5 & $(41.4-51.7)$ \\
\hline Medical-Surgical & 127 & 12.1 & $(10.2-14.1)$ & 18 & 14.2 & $(8.1-20.2)$ & 31 & 24.4 & $(16.9-31.9)$ & 78 & 61.4 & $(53.0-69.9)$ \\
\hline Medical & 559 & 53.4 & $(50.4-56.4)$ & 122 & 21.9 & $(18.4-25.2)$ & 197 & 35.2 & $(31.3-39.2)$ & 240 & 42.9 & $(38.8-47.0)$ \\
\hline
\end{tabular}


Table 2. Continued

\begin{tabular}{|c|c|c|c|c|c|c|c|c|c|c|c|c|}
\hline & \multicolumn{3}{|c|}{ Overall } & \multicolumn{3}{|c|}{ Current smokers } & \multicolumn{3}{|c|}{ Former smokers } & \multicolumn{3}{|c|}{ Von smokers } \\
\hline & n & $o_{0}$ & $95^{\circ} \circ \mathrm{CI}$ & n & $0^{2}$ & $95^{\circ} \circ \mathrm{OI}$ & n & $o^{2}$ & $95^{\circ} \circ \mathrm{CI}$ & n & $\%^{2}$ & $95^{\circ} \% \mathrm{CI}$ \\
\hline \multicolumn{13}{|c|}{ Number of beds } \\
\hline$\leq 300$ & 541 & 51.7 & $(48.6-54.7)$ & 111 & 20.5 & $(17.1-23.9)$ & 176 & 32.5 & $(28.6-36.5)$ & 254 & 47.0 & $(42.7-51.2)$ \\
\hline$>300$ & 506 & 48.3 & $(45.3-51.4)$ & 104 & 20.6 & $(17.0-24.1)$ & 170 & 33.6 & $(29.5-37.7)$ & 232 & 45.8 & $(41.5-50.2)$ \\
\hline \multicolumn{13}{|c|}{ HP smoking prevalence } \\
\hline$<30 \%$ & 801 & 76.5 & $(73.9-79.1)$ & 160 & 20.0 & $(17.2-22.7)$ & 265 & 33.1 & $(29.8-36.3)$ & 376 & 46.9 & $(43.5-50.4)$ \\
\hline$\geq 30 \%$ & 246 & 23.5 & $(20.9-26.1)$ & 55 & 22.4 & $(17.2-27.6)$ & 81 & 32.9 & $(27.1-38.8)$ & 110 & 44.7 & $(38.5-50.9)$ \\
\hline \multicolumn{13}{|c|}{ Smoking cessation program } \\
\hline Yes & 813 & 77.7 & $(75.1-80.2)$ & 165 & 20.3 & $(17.5-23.1)$ & 273 & 33.6 & $(30.3-36.8)$ & 375 & 46.1 & $(42.7-49.6)$ \\
\hline No & 234 & 22.3 & $(19.8-24.9)$ & 50 & 21.4 & $(16.1-26.6)$ & 73 & 31.2 & $(25.3-37.1)$ & 111 & 47.4 & $(41.0-53.8)$ \\
\hline \multicolumn{13}{|c|}{ Accreditation level } \\
\hline Gold & 337 & 32.2 & $(29.4-35.0)$ & 69 & 20.5 & $(16.2-24.8)$ & 126 & 37.4 & $(32.2-42.6)$ & 142 & 42.1 & $(36.9-47.4)$ \\
\hline Silver & 317 & 30.3 & $(27.5-33.1)$ & 63 & 19.9 & $(15.5-24.3)$ & 91 & 28.7 & $(23.7-33.7)$ & 163 & 51.4 & $(45.9-56.9)$ \\
\hline Bronze & 147 & 14.0 & $(11.9-16.1)$ & 28 & 19.0 & $(12.7-25.4)$ & 48 & 32.7 & $(25.1-40.2)$ & 71 & 48.3 & $(40.2-56.4)$ \\
\hline Member & 246 & 23.5 & $(20.9-26.1)$ & 55 & 22.4 & $(17.2-27.6)$ & 81 & 32.9 & $(27.1-38.8)$ & 110 & 44.7 & $(38.5-50.9)$ \\
\hline
\end{tabular}

HP: health professionals. 1 Percentage per column. 2 Percentage per row.

smoking prevalence was observed among medicalsurgical wards. In the bivariate analysis, smoking prevalence was not significantly related to any of the explanatory variables we assessed that represented hospital characteristics.

\section{Smoking patterns among daily smokers before and during hospitalization}

Among all smokers, 97.2\% were daily smokers (209/215). The majority of male smokers were 4564 years (49.6\%, 95 CI\%: 41.3-58.0); the majority of female smokers were $\leqslant 45$ years $(55.7 \%, 95$ CI\%: 44.1-67.4). Both men and women smoked mainly manufactured cigarettes, but nearly one-quarter smoked RYO cigarettes. Other tobacco products (cigars, pipes, and e-cigarettes) were consumed based on anecdotal reports. The majority of smokers consumed exclusively manufactured cigarettes (75.6\%), but $12.7 \%$ consumed only RYOs, and $11.7 \%$ combined these two tobacco products (Table 3 ).

Before hospitalization, $44.1 \%$ of daily smokers consumed 10-19 CPD, and 72.7\% consumed their first cigarette within the first 30 min of waking. Thus, $44.9 \%$ of smokers had an intermediate level of nicotine dependence according to the HSI. High levels of nicotine dependence were more frequently observed among male smokers than among female smokers, but the difference was not statistically significant (Table 3).
A total $75.7 \%$ of daily smokers expressed their wish to quit smoking, if it could be done easily during hospitalization. Among all smokers, 38.1\% had high interest in quitting now, and $21.3 \%$ had no interest in quitting. Men were more interested in quitting than women (Table 3). Among daily smokers, $24.2 \%$ admitted to consuming tobacco during hospitalization, and the proportion was similar between men and women. When analysing their length of stay and smoking during hospitalization, we observed that $31.6 \%$ of smokers with 1-day stay smoked during hospitalization versus $19.8 \%$ of those who were interviewed in their 2 nd to 5 th day of stay, and $26.5 \%$ of those with $>5$ days of stay $(p=0.417)$. The average number of cigarettes consumed per day was 3 (range $=1$ to 20 ). Smokers who consumed tobacco did it $15.5 \%$ of the times inside the hospital (in their room, wc, stairs, terraces), $63.5 \%$ in outdoor areas belonging to the hospital (entrances, gardens, parkings, etc), and $21.0 \%$ outside the perimeter of the hospital. Smokers who consumed tobacco during hospitalization did not differ from those who remained abstinent from smoking, based on sex, age, nicotine dependence, and whether the hospital provided smoking cessation services. The percentage of smokers with exhaled carbon monoxide levels $>6$ ppm was higher among male smokers than among female smokers $(63.0 \%$ vs $48.2 \%, p=0.069)$ It is known, that smokers who remain abstinent for 
Table 3. Patterns of daily tobacco use, before and during hospitalization (Hospitals of Barcelona Province, 2014-2015)

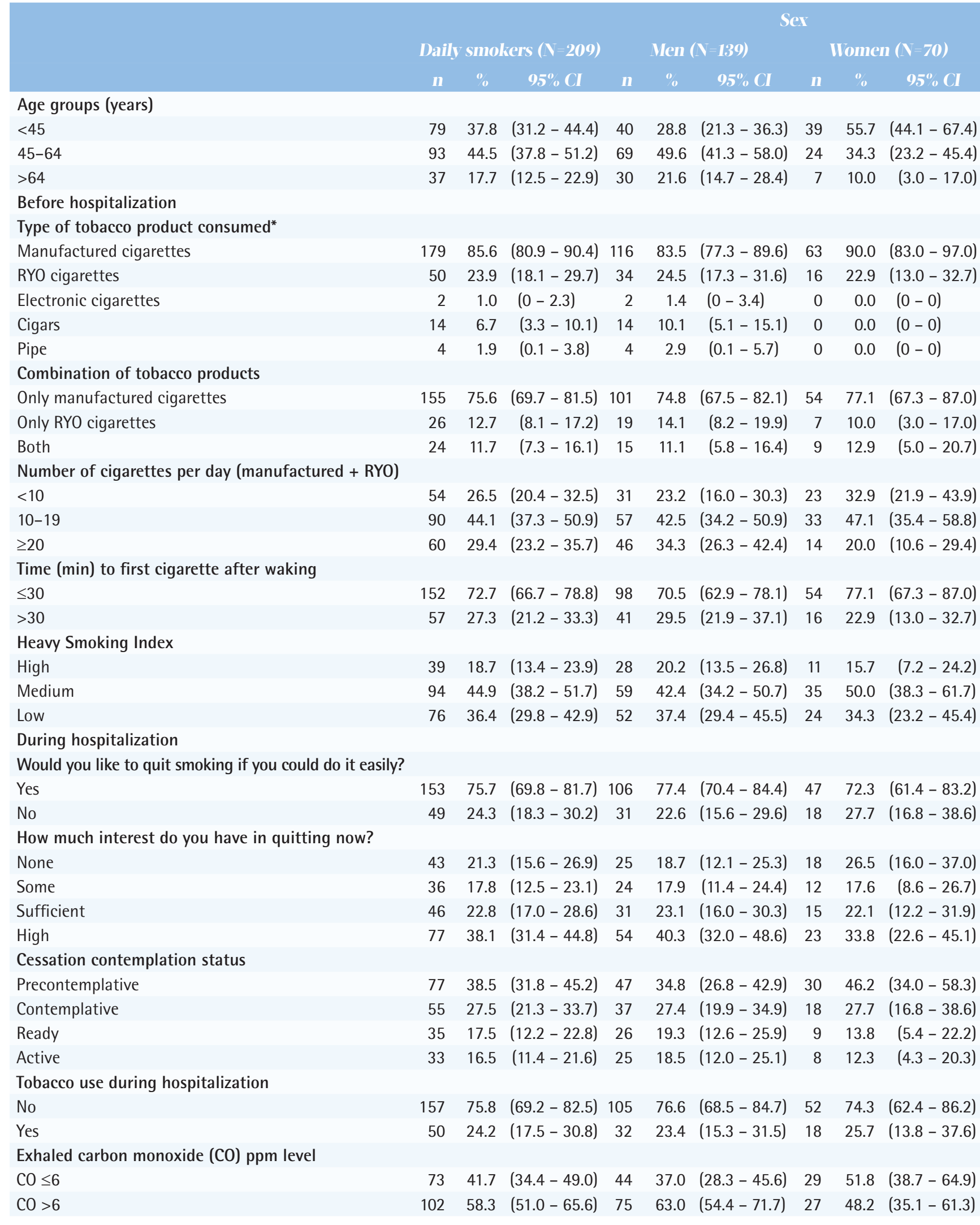

* Multiple responses allowed. RYO: roll your own cigarettes. 
more than 6 days might have lower CO levels ${ }^{22}$. Our results showed that $73.9 \%$ of smokers (with more than 5 days stay) who admitted to having smoked during hospitalization had a positive $\mathrm{CO}(>6 \mathrm{ppm})$ in contrast to $34.9 \%$ of those who remained abstinent $(\mathrm{p}=0.001)$.

\section{Smoking patterns and sociodemographic characteristics of former smokers}

Male former smokers were mostly $>64$ years, and female former smokers were significantly younger (45-64 years; $p \leqslant 0.001$, Table 4 ). Former smokers were more likely to have smoked daily, rather than occasionally, but a higher percentage of women than men had smoked occasionally. Among former daily smokers, we observed differences between the sexes. For example, the majority of men previously consumed $\geqslant 20$ CPD (70.9\%, 95\% CI: 64.7-77.1) and a minority of women previously consumed $\geqslant 20 \mathrm{CPD}$ (37.5\%, 95\% CI: 25.6-49.4; p<0.05). About 74.1\%

Table 4. Smoking pattern and sociodemographic characteristics among former smokers $(\mathrm{n}=346)$ (Hospitals of Barcelona Province, 2014-2015)

\begin{tabular}{|c|c|c|c|c|c|c|c|c|c|}
\hline \multirow{4}{*}{ Age groups (years) } & \multicolumn{9}{|c|}{ Ser } \\
\hline & \multicolumn{3}{|c|}{ Former smoker } & \multicolumn{3}{|c|}{ Ven } & \multicolumn{3}{|c|}{ Women } \\
\hline & n & $\%$ & $95^{\circ} \% \mathrm{CI}$ & \multirow[t]{2}{*}{ n } & $\%$ & \multirow[t]{2}{*}{$95^{\circ} \% \mathrm{CI}$} & \multirow[t]{2}{*}{ n } & $\%$ & \multirow[t]{2}{*}{$95^{\circ} \% \mathrm{CI}$} \\
\hline & & & & & & & & & \\
\hline$<45$ & 49 & 14.2 & $(10.5-17.8)$ & 17 & 6.8 & $(3.7-9.9)$ & 32 & 33.7 & $(24.2-43.2)$ \\
\hline $45-64$ & 122 & 35.3 & $(30.2-40.3)$ & 81 & 32.3 & $(26.5-38.1)$ & 41 & 43.1 & $(33.2-53.1)$ \\
\hline$>64$ & 175 & 50.5 & $(45.3-55.8)$ & 153 & 60.9 & $(54.9-67.0)$ & 22 & 23.2 & $(14.7-31.6)$ \\
\hline \multicolumn{10}{|l|}{ Daily consumption } \\
\hline No (occasionals) & 47 & 13.6 & $(9.7-17.5)$ & 20 & 8.0 & $(4.5-11.5)$ & 27 & 28.4 & $(17.7-39.1)$ \\
\hline Yes & 299 & 86.4 & $(82.5-90.3)$ & 231 & 92.0 & $(88.5-95.5)$ & 68 & 71.6 & $(60.9-82.3)$ \\
\hline \multicolumn{10}{|c|}{ Number of cigarettes per day (manufactured + RYO) * } \\
\hline$<10$ & 40 & 14.8 & $(10.6-19.1)$ & 19 & 9.2 & $(5.3-13.2)$ & 21 & 32.8 & $(21.3-44.3)$ \\
\hline $10-19$ & 60 & 22.2 & $(17.3-27.2)$ & 41 & 19.9 & $(14.5-25.4)$ & 19 & 29.7 & $(18.5-40.9)$ \\
\hline$\geq 20$ & 170 & 63.0 & $(57.2-68.7)$ & 146 & 70.9 & $(64.7-77.1)$ & 24 & 37.5 & $(25.6-49.4)$ \\
\hline \multicolumn{10}{|l|}{ Number of quit attempts } \\
\hline $1-2$ & 253 & 74.1 & $(69.5-78.8)$ & 178 & 72.0 & $(66.5-77.7)$ & 75 & 79.8 & $(71.7-87.9)$ \\
\hline $3-5$ & 66 & 19.4 & $(15.2-23.5)$ & 52 & 21.1 & $(16.0-26.1)$ & 14 & 14.9 & $(7.7-22.1)$ \\
\hline$\geq 6$ & 22 & 6.5 & $(3.8-9.1)$ & 17 & 6.9 & $(3.7-10.0)$ & 5 & 5.3 & $(0.8-9.9)$ \\
\hline \multicolumn{10}{|l|}{ Reasons for quitting ** } \\
\hline Doctor/nurse recommendation & 110 & 31.8 & $(26.9-36.7)$ & 93 & 26.9 & $(22.2-31.5)$ & 17 & 4.9 & $(2.6-7.2)$ \\
\hline Tobacco use annoyances & 134 & 38.7 & $(33.6-43.9)$ & 118 & 34.1 & $(29.1-39.1)$ & 16 & 4.6 & $(2.4-6.8)$ \\
\hline Health concerns & 175 & 50.6 & $(45.3-55.8)$ & 141 & 40.8 & $(35.6-45.9)$ & 34 & 9.8 & $(6.7-13.0)$ \\
\hline Decrease of my physical performance & 75 & 21.7 & $(17.3-26.0)$ & 66 & 19.1 & $(14.9-23.2)$ & 9 & 2.6 & $(0.9-4.3)$ \\
\hline Familial pressure & 71 & 20.5 & $(16.3-24.8)$ & 47 & 13.6 & $(10.0-17.2)$ & 24 & 6.9 & $(4.3-9.6)$ \\
\hline Personal decision & 215 & 62.1 & $(57.0-67.2)$ & 154 & 44.5 & $(39.3-49.7)$ & 61 & 17.6 & $(13.6-21.6)$ \\
\hline Economic reasons & 21 & 6.1 & $(3.6-8.6)$ & 14 & 4.0 & $(2.0-6.1)$ & 7 & 2.0 & $(0.5-3.5)$ \\
\hline Health problems (tobacco-related diseases) & 15 & 4.3 & $(2.2-6.5)$ & 12 & 3.5 & $(1.5-5.4)$ & 3 & 0.9 & $(0-1.8)$ \\
\hline Other reasons & 35 & 10.1 & $(6.9-13.3)$ & 20 & 5.8 & $(3.3-8.2)$ & 15 & 4.3 & $(2.2-6.5)$ \\
\hline \multicolumn{10}{|l|}{ Resources used to quit smoking ${ }^{* *}$} \\
\hline Professional healthcare & 31 & 9.0 & $(6.0-12.0)$ & 26 & 7.5 & $(4.7-10.3)$ & 5 & 1.4 & $(0.2-2.7)$ \\
\hline Use of e-health technologies (apps. webs) & 0 & 0.0 & $(0-0)$ & 0 & 0.0 & $(0-0)$ & 0 & 0.0 & $(0-0)$ \\
\hline Self-help book & 7 & 2.0 & $(0.5-3.5)$ & 5 & 1.4 & $(0.2-2.7)$ & 2 & 0.6 & $(0-1.4)$ \\
\hline Other & 7 & 2.0 & $(0.5-3.5)$ & 6 & 1.7 & $(0.4-3.1)$ & 1 & 0.3 & $(0-0.9)$ \\
\hline None & 302 & 87.3 & $(83.8-90.8)$ & 216 & 62.4 & $(57.3-67.5)$ & 86 & 24.9 & $(20.3-29.4)$ \\
\hline \multicolumn{10}{|l|}{ Pharmacological treatment used } \\
\hline Yes & 46 & 13.5 & $(9.9-17.2)$ & 36 & 14.6 & $(10.2-19.1)$ & 10 & 10.6 & $(4.4-16.9)$ \\
\hline No & 294 & 86.5 & $(82.8-90.1)$ & 210 & 85.4 & $(80.9-89.8)$ & 84 & 89.4 & $(83.1-95.6)$ \\
\hline
\end{tabular}


of former smokers had attempted to quit 1 or 2 times before finally quitting. The two most reported reasons for quitting were their own personal decision and health concerns. Among former smokers, 87.3\% did not use any resources to assist them in quitting, and $13.5 \%$ used a pharmacological treatment to quit (Table 4).

\section{Predictors associated with smokers and former smokers}

Table 5 displays the aOR (adjusted for sex and age) that a current smoker or a former smoker would exhibit characteristics represented by patient-related and hospital-related explanatory variables. Current smokers were more likely to be young men. Tobacco consumption was more likely $(\mathrm{aOR}=2.76)$ to be observed among patients with primary or less than primary education levels, compared to patients with a university degree. No other significant relationship emerged between smoking and any of the hospitalrelated variables studied.

The odds ratio of being a former smoker was higher among men $(\mathrm{aOR}=5.85)$ compared to women. Smokers were more likely to be 30-69 years old, rather than $>70$ years old. Former smokers were most frequently patients with a university degree and patients that had retired from working. Patients admitted to medical-surgical wards were more likely to be former smokers than those in surgical wards $(\mathrm{aOR}=2.39)$.

Table 5. Univariate and multivariate models of a current smoker or former smoker (Hospitals of Barcelona Province, 2014-2015)

\begin{tabular}{|c|c|c|c|c|c|c|c|c|}
\hline \multirow[b]{2}{*}{ Descriptive variables } & \multicolumn{4}{|c|}{ Current smoker } & \multicolumn{4}{|c|}{ Former smoker } \\
\hline & cOR & $95^{\circ} \circ \mathrm{CI}$ & $\mathrm{aOR}$ & $95^{\circ} \% \mathrm{CI}$ & COR & $95 \% \mathrm{CI}$ & $\mathrm{aOR}$ & $95^{\circ} \% \mathrm{CI}$ \\
\hline \multicolumn{9}{|c|}{ Patient related characteristics } \\
\hline \multicolumn{9}{|l|}{ Sex } \\
\hline Male & 7.22 & $(4.88-10.68)$ & 7.47 & $(4.88-11.43)$ & 7.11 & $(5.15-9.80)$ & 5.85 & $(4.16-8.22)$ \\
\hline Female & 1.00 & & 1.00 & & 1.00 & & 1.00 & \\
\hline \multicolumn{9}{|l|}{ Age groups (years) } \\
\hline $18-29$ & 7.56 & $(3.63-15.77)$ & 8.24 & $(3.25-20.91)$ & 0.48 & $(0.21-1.06)$ & 0.74 & $(0.30-1.84)$ \\
\hline $30-39$ & 11.07 & $(5.69-21.54)$ & 11.55 & $(4.84-27.55)$ & 1.46 & $(0.85-2.52)$ & 1.63 & $(0.78-3.38)$ \\
\hline $40-49$ & 13.32 & $(7.05-25.17)$ & 11.48 & $(5.10-25.82)$ & 1.22 & $(0.70-2.11)$ & 1.41 & $(0.70-2.85)$ \\
\hline $50-59$ & 11.76 & $(6.23-22.20)$ & 10.01 & $(4.60-21.81)$ & 2.35 & $(1.44-3.81)$ & 2.83 & $(1.52-5.29)$ \\
\hline $60-69$ & 3.50 & $(1.87-6.54)$ & 3.70 & $(1.93-7.09)$ & 1.47 & $(0.98-2.22)$ & 1.58 & $(1.03-2.43)$ \\
\hline$>70$ & 1.00 & & 1.00 & & 1.00 & & 1.00 & \\
\hline \multicolumn{9}{|l|}{ Education } \\
\hline Primary or less & 2.97 & $(1.61-5.49)$ & 2.76 & $(1.44-5.28)$ & 0.73 & $(0.45-1.18)$ & 0.75 & $(0.46-1.24)$ \\
\hline High School & 1.88 & $(0.98-3.61)$ & 1.45 & $(0.73-2.89)$ & 0.76 & $(0.44-1.30)$ & 0.73 & $(0.42-1.25)$ \\
\hline University & 1.00 & & 1.00 & & 1.00 & & 1.00 & \\
\hline \multicolumn{9}{|l|}{ Occupation } \\
\hline Unemployed & 1.21 & $(0.65-2.24)$ & 1.16 & $(0.59-2.29)$ & 0.74 & $(0.37-1.50)$ & 0.93 & $(0.45-1.91)$ \\
\hline Retired & 1.24 & $(0.69-2.21)$ & 0.96 & $(0.52-1.78)$ & 1.33 & $(0.77-2.27)$ & 1.51 & $(0.87-2.62)$ \\
\hline Other & 0.34 & $(0.15-0.78)$ & 0.29 & $(0.12-0.67)$ & 0.30 & $(0.14-0.65)$ & 0.35 & $(0.16-0.77)$ \\
\hline Employed & 1.00 & & 1.00 & & 1.00 & & 1.00 & \\
\hline \multicolumn{9}{|l|}{ Partner's smoking status } \\
\hline No partner & 2.18 & $(1.40-3.39)$ & 2.07 & $(1.31-3.27)$ & 0.84 & $(0.58-1.21)$ & 0.89 & $(0.61-1.29)$ \\
\hline Smoker partner & 5.75 & $(3.48-9.52)$ & 6.01 & $(3.57-10.11)$ & 1.53 & $(0.94-2.48)$ & 1.50 & $(0.91-2.47)$ \\
\hline Non-smoker partner & 1.00 & & 1.00 & & 1.00 & & 1.00 & \\
\hline \multicolumn{9}{|l|}{ Perceived health status } \\
\hline Excellent/Very good & 1.76 & $(1.00-3.07)$ & & & 1.67 & $(1.00-2.79)$ & & \\
\hline Good & 1.30 & $(0.78-2.18)$ & & & 1.30 & $(0.79-2.12)$ & & \\
\hline Adequate/Poor & 1.00 & & & & 1.00 & & & \\
\hline \multicolumn{9}{|l|}{ Barthel Index } \\
\hline Independent (100) & 0.93 & $(0.54-1.57)$ & & & 0.86 & $(0.59-1.26)$ & & \\
\hline Dependent $(<100)$ & 1.00 & & & & 1.00 & & & \\
\hline
\end{tabular}


Table 5. Continued

\begin{tabular}{|c|c|c|c|c|c|c|c|c|}
\hline \multirow[b]{2}{*}{ Descriptive variables } & \multicolumn{4}{|c|}{ Current smoker } & \multicolumn{4}{|c|}{ Former smoker } \\
\hline & cOR & $95 \% \mathrm{CI}$ & aOR & $95^{\circ} \mathrm{OCI}$ & cOR & $95^{\circ} \circ \mathrm{CI}$ & aOR & $95^{\circ} \% \mathrm{CI}$ \\
\hline \multicolumn{9}{|c|}{ Hospital related characteristics } \\
\hline \multicolumn{9}{|l|}{ Level of center } \\
\hline General hospital & 1.00 & & & & 1.00 & & & \\
\hline High-technology hospital & 0.76 & $(0.50-1.16)$ & & & 1.05 & $(0.73-1.51)$ & & \\
\hline \multicolumn{9}{|l|}{ Type of ward } \\
\hline Surgical & 1.00 & & & & 1.00 & & & \\
\hline Medical-Surgical & 0.58 & $(0.29-1.18)$ & & & 2.39 & $(1.26-4.54)$ & & \\
\hline Medical & 1.09 & $(0.74-1.63)$ & & & 1.19 & $(0.85-1.67)$ & & \\
\hline \multicolumn{9}{|l|}{ Number of beds } \\
\hline$\leq 300$ & 1.00 & & & & 1.00 & & & \\
\hline$>300$ & 1.00 & $(0.70-1.44)$ & & & 0.98 & $(0.72-1.34)$ & & \\
\hline \multicolumn{9}{|l|}{ HP smoking prevalence } \\
\hline$<30 \%$ & 1.00 & & & & 1.00 & & & \\
\hline$\geq 30 \%$ & 1.51 & $(0.98-2.32)$ & & & 1.01 & $(0.70-1.46)$ & & \\
\hline \multicolumn{9}{|l|}{ Smoking cessation program } \\
\hline Yes & 1.00 & & & & 1.00 & & & \\
\hline No & 1.11 & $(0.72-1.72)$ & & & 0.82 & $(0.57-1.19)$ & & \\
\hline \multicolumn{9}{|l|}{ Accreditation level } \\
\hline Gold & 1.00 & & & & 1.00 & & & \\
\hline Silver & 0.75 & $(0.47-1.19)$ & & & 0.75 & $(0.50-1.11)$ & & \\
\hline Bronze & 0.74 & $(0.41-1.34)$ & & & 0.74 & $(0.46-1.21)$ & & \\
\hline Member & 1.27 & $(0.78-2.09)$ & & & 0.86 & $(0.57-1.30)$ & & \\
\hline
\end{tabular}

HP: health professional, cOR: odds ratio adjusted for age and sex, aOR: odds ratio fully adjusted for all the variables.

\section{DISCUSSION}

This study showed that smoking prevalence was high among hospitalized patients. Our findings indicate that hospitalized smokers comprised mainly males that were $<64$ years old, had primary or less than primary education, and had a partner with a smoking habit. The majority of smokers exhibited an intermediate nicotine dependence, and were mainly in precontemplative or contemplative stages of quitting. A quarter of smokers consumed tobacco during their hospital stay.

Compared to the Catalan Health Survey (ESCA) results from $2014^{24}$, adult hospitalized patients (>18 years) showed a lower overall prevalence of smokers than the general population older than 15 years (20.5\% vs $25.9 \%)$. Nonetheless, this might be due to the mean age of our study sample (hospitalized patients), which corresponded to an aging population. In fact, when comparing age groups, the overall prevalence of current smokers in the middle age group (45-64 years; $30.6 \%$ ) was similar to that of the Catalan population between $45-54$ years $(30.5 \%)$, but higher than the Catalan population between $55-65$ years $(21.9 \%)^{24}$. When comparing sex and age groups, our study found that $47.2 \%$ of men $<45$ years were current smokers; in the Catalan population, the smoking prevalence was lower among men between $35-44$ years $(35.2 \%)$ and higher among men between $25-34$ years $(50.0 \%)$. Again, no differences were found between our sample and the Catalan population for individuals $>65$ years (men $12.4 \%$ vs $10.9 \%$; women $3.1 \%$ vs $4.5 \%$, respectively $)^{24}$. These results suggest that the prevalence of smokers among hospitalized patients was not different from that of the general population. This finding indicates that there is an opportunity for health care services to intervene in young patients that are not chronically ill, when they are treated in acute-care hospitals.

Only two previous studies have monitored smoking consumption in hospitalized populations in Catalonia. The earlier study found a higher overall smoking prevalence $(27.8 \%$ in 2002 and $30.7 \%$ in 2004$)^{25}$, and a later study found a slightly lower smoking prevalence $(18.8 \% \text { in } 2006)^{26}$, compared to the overall prevalence reported 
here $(20.5 \%)$. These differences might be due to the differences in timing or design; the earlier studies were conducted 10 years ago and only in one center. In addition, the earlier investigations could have carried some selection bias, because not all hospital wards were included in the sampling strategies. This study is the first to monitor tobacco consumption among hospitalized patients from all wards in acute-care hospitals in our region. In the United States, a recent study that included all patients admitted to a General Hospital over 3 years (2007 to 2010 ) concluded that $21.1 \%$ of patients were smokers, and $18.4 \%$ had smoked during their hospital stay ${ }^{11}$. However, although our prevalence of tobacco consumption was similar, we found lower compliance with the smoke-free law than that found by Regan and colleagues ${ }^{11}$. This fact raised two safety concerns in our region. First, smoking may have direct, negative consequences that could delay recovery; and second, smoking could cause a fire, putting others in danger in the complicated context of a hospital. Consequently, hospitals in Catalonia should improve their communications about the smoke-free campus policy and about the risks of smoking during hospitalization. In addition, hospitals should monitor the implementation of the smoke-free compliance on a yearly basis.

In Catalonia, there are around 972995 annual hospitalizations ${ }^{27}$. According to our study, 20.5\% of acute-care patients smoked, and of these, the majority expressed some or a high interest in quitting. Extending these proportions to all hospitalizations suggests that about 157000 hospitalized smokers might be motivated to quit annually. In addition, as mentioned above, our profile of smokers in general hospitals indicates mainly young men. Thus, our data supports the notion that hospitalization could serve as a promising window of opportunity for approaching smokers and encouraging cessation ${ }^{28}$. This opportunity has the advantages, in the context of a comprehensive smoke-free hospital, that smokers are obliged to abstain at least temporarily from tobacco use and that they are in regular contact with health professionals. A previous study found that between $60-70 \%$ of inpatients had attempted to quit smoking while they were hospitalized ${ }^{29}$. In the present study, about three-quarters of smokers expressed a wish to quit smoking, and about onethird was ready to undertake an attempt. Therefore, hospitalization could provide a unique opportunity for identifying and engaging smokers, initiating cessation treatments, and facilitating appropriate follow-up and support procedures ${ }^{30}$.

A previous meta-analysis showed that smoking cessation programs were effective when they began during a hospital stay, regardless of the reason for admission, and when nicotine replacement therapy (NRT) was offered and a follow-up visit was provided at one month after discharge ${ }^{30}$. Therefore, health providers, should offer effective smoking cessation assistance to hospitalized smokers, regardless of the diagnosis or hospital unit, as shown in our study ${ }^{3}$.

Internationally, several health organizations have adopted the 5As intervention model for smoking cessation proposed by evidence-based guidelines ${ }^{31}$. This model is based on five steps: 1) Ask patients about smoking at every visit; 2) Advise all tobacco users to quit; 3 ) Assess smoker's willingness to try to quit; 4) Assist smoker's efforts with treatment and referrals; and 5) Arrange follow-up contacts to support cessation efforts ${ }^{31}$. However, deficiencies persist in implementing smoking cessation interventions as part of routine practices in hospital settings ${ }^{32}$. One situation recognized to render smoking cessation interventions suboptimal is when the health professional is a smoker ${ }^{33,34}$. For instance, it was found that nurses that smoked were less likely to advise their patients to quit and less willing to arrange smoking cessation follow-up appointments ${ }^{34}$. In addition, a recent study conducted among health professionals in Catalonia showed that the clinical healthcare workers did not perform the 5 As completely ${ }^{35}$. The main barriers were: smoking cessation was considered not part of their job, lack of familiarity with practical guidelines, lack of previous positive experiences, and lack of organizational support ${ }^{35}$.

To improve smoking cessation interventions, several hospitals have taken numerous actions, including providing training to health professionals and requesting compulsory performance indicators ${ }^{30,36,37}$. Training has been strongly associated with higher levels of confidence, more frequent interventions, and fewer barriers to 
providing cessation services ${ }^{38}$.

In Catalonia, several actions have been undertaken to implement tobacco control interventions in hospitals, beyond the legislative framework ${ }^{39}$. In 2000 , the XCHsF promoted a 'tobacco control hospital model' that implemented organizational and cultural changes ${ }^{39}$. This model required the organization to make a commitment to adopting, integrally and progressively, a series of ten standards. This organizational change involved creating a policy working group that was integrated into the hospital management team and included key individuals in the institution (champions). This working group was responsible for designing the tobacco-control policy, scaling it down, and ensuring that it was properly communicated, monitored, and evaluated ${ }^{38}$. Thus, the working group must clearly communicate the policies to the other staff members, the patients, and the community. Currently, the tobacco control champions have shown extraordinary interest in the adoption, implementation, and evaluation of activities ${ }^{40,41}$. Because training is a key factor in the sustainability of these programs, the $\mathrm{XCHsF}$ has offered in-person and online training, which takes place every year and aims to reach a broad number of hospital workers ${ }^{42}$.

The large prevalence of smokers with medium and high nicotine dependences (63.6\% of smokers) has provided a strong rationale for offering drug treatment, such as nicotine replacement treatment (NRT). Currently, the majority of Catalan hospitals have implemented this therapeutic aid ${ }^{40}$. Clearly, the impact of these measures on increasing health care professionals's interventions in providing tobacco cessation, patient smoking prevalence and cessation rates should be tested in future studies.

\section{Limitations}

This study has several limitations. First, it is a crosssectional survey, and thus, our results cannot lead to any conclusions about direct causal effects; instead, our results only indicate associations. Second, this study relied on self-reported responses; however, tobacco consumption was verified with a COoximeter. Third, we excluded patients admitted into emergency units and critical care units; thus, our sample might not be representative of all patients admitted into Catalan hospitals. In cases where admission to those units was related to smoking, omission of these data could lead to a bias in our results towards a lower prevalence of smoking. However, we implemented this exclusion criterion to assure the validity of our information, because all our patients were conscious in space and time. Fourth, due to our selection of hospitals by convenience, we could have introduced a selection bias, because it is possible that we chose only hospitals with the most interest in smoking cessation practices. However, that bias would have led to an underestimation of the current smoking problem in Catalan hospitals in this study. Nonetheless, this study was the first in Spain to explore smoking prevalence among hospitalized patients in multiple hospitals and units, with a large, representative sample size.

\section{CONCLUSIONS}

Overall, $20.5 \%$ of hospitalized patients were current smokers, over $60 \%$ of smokers had medium or high nicotine dependences, and one-quarter smoked during hospitalization. Moreover, we found that about $75.7 \%$ of smokers expressed a wish to quit smoking, and half of them were ready or in a complentative stage to undertake an attempt. Our findings indicate that there is a need to initiate smoking cessation interventions among patients in all units and service areas during hospitalization. It is necessary to provide adequate therapeutic support and routine monitoring of patient cravings for cigarettes. The implementation of cessation interventions could avoid infringements of smoke-free policies and improve patient safety, hospital efficiency, and clinical outcomes. Future investigations should focus on testing the effectiveness of smoke-free policies combined with smoking cessation interventions initiated in hospitals. In addition, our findings indicate that these interventions should mainly focus on young patients that are not chronically ill. This study suggests that hospitalization represents a promising window for initiating smoking interventions addressed to all patients, particularly for patients who are men and without chronic diseases. Finally, health administrations, hospitals, and community services should work together to facilitate the initiation of smoking cessation treatments for 
patients admitted to smoke-free hospitals, especially after applying smoke-free campus bans.

\section{REFERENCES}

1. World Health Organization. WHO report on the global tobacco epidemic. 2013.

2. WHO Tobacco Free Initiative, ed. 2012 global progress report on implementation of the WHO framework convention on tobacco control. Geneva: WHO; 2012.

3. WHO Tobacco Free Iniciative, ed. The role of health professionals in tobacco control. Geneva: WHO; 2012.

4. Longo DR, Feldman MM, Kruse RL, Brownson RC, Petroski GF, Hewett JE. Implementing smoking bans in American hospitals: results of a national survey. Tob Control. 1998;7(1):47-55. doi:10.1136/tc.7.1.47

5. Longo DR, Brownson RC, Johnson JC, et al. Hospital smoking bans and employee smoking behavior: Results of a national survey. JAMA. 1996;275(16):1252-1257. doi:10.1001/jama.1996.03530400040036

6. Martinez C, Garcia M, Mendez E, Peris M, Fernandez E. Barriers and challenges for tobacco control in a smoke-free hospital. Cancer Nurs. 2008;31(2):88-94. doi:10.1097/01.ncc.0000305708.37530.ee

7. Mullen KA, Coyle D, Manuel D, et al. Economic evaluation of a hospital-initiated intervention for smokers with chronic disease, in Ontario, Canada. Tob Control. 2014:24. doi:10.1136/tobaccocontrol-2013-051483

8. Rigotti NA, Munafo MR, Stead LF. Smoking cessation interventions for hospitalized smokers: A systematic review. Arch Intern Med. 2008;168(18):1950-1960. doi:10.1001/archinte.168.18.1950

9. Rigotti NA, Arnsten JH, McKool KM, Wood-Reid KM, Pasternak RC, Singer DE. Smoking by patients in a smoke-free hospital: Prevalence, predictors, and implications. Prev Med. 2000;31(2):159-166. doi:10.1006/pmed.2000.0695

10. Ratschen E, Britton J, McNeill A. Smoke-free hospitals the english experience: Results from a survey, interviews, and site visits. BMC Health Serv Res. 2008;8:41. doi:10.1186/1472-6963-8-41

11. Regan S, Viana JC, Reyen M, Rigotti NA. Prevalence and predictors of smoking by inpatients during a hospital stay. Arch Intern Med. 2012;172(21):1670-1674. doi:10.1001/2013.jamainternmed.300

12. Bilal U, Fernandez E, Beltran P, Navas-Acien A, Bolumar F, Franco M. Validation of a method for reconstructing historical rates of smoking prevalence. Am J Epidemiol. 2014;179(1):15-19. doi:10.1093/aje/kwt224

13. Ministerio de Sanidad y Consumo, ed. Encuesta nacional de salud de españa 1987, 1993, 1995, 1997, 2001 y 2003. http://www.msc.es/estadEstudios/estadisticas/ en. Published, 2003. Accessed April 11, 2018.

14. Ministerio de Sanidad, Servicios Sociales e Igualdad y Instituto Nacional de Estadística. Encuesta nacional de salud de españa 2011/12 (ENSE 2011/12). http://www. msc.es/estadEstudios/estadisticas/encuestaNacional/ encuesta2011. Accessed June 3, 2013.

15. Gutierrez-Abejon E, Rejas-Gutierrez J, Criado-Espegel P, Campo-Ortega EP, Brenas-Villalon MT, MartinSobrino N. Smoking impact on mortality in Spain in 2012. Med Clin (Barc). 2015;145(12):520-525. doi:10.1016/j.medcle.2016.04.020

16. Instituto Nacional de Estadistica. Encuesta de morbilidad hospitalaria. http://www.ine.es/prensa/np762.pdf. Updated, 2013. Accessed August 27, 2013.

17. Martinez C, Martinez-Sanchez JM, Robinson G, Bethke C, Fernandez E. Protection from secondhand smoke in countries belonging to the WHO European Region: An assessment of legislation. Tob Control. 2014;23(5):403411. doi:10.1136/tobaccocontrol-2012-050715

18. Ley $42 / 2010$, por la que se modifica la Ley $28 / 2005$, de 26 de diciembre, de medidas sanitarias frente al tabaquismo y reguladora de la venta, el suministro, el consumo y la publicidad de los productos del tabaco. BOE 318:109188 - 109194B. http://www.boe.es/ boe/dias/2010/12/31/pdfs/BOE-A-2010-20138.pdf. Published December 31, 2010. Accessed, 2011.

19. Departament de Salut. Generalitat de Catalunya. Enquesta de Salut de Catalunya 2006.

20. World Health Organization, ed. The evaluation and monitoring of public action on tobacco. Smoke-free Europe. Copenhagen: World Health Organization; 1990.

21. Chabrol H, Niezborala M, Chastan E, de Leon J. Comparison of the heavy smoking index and of the Fagerstrom test for nicotine dependence in a sample of 749 cigarette smokers. Addict Behav. 2005;30(7):14741477. doi:10.1016/j.addbeh.2005.02.001

22. Middleton ET, Morice AH. Breath carbon monoxide as an indication of smoking habit. Chest. 2000;117(3):758763. doi:10.1378/chest.117.3.758

23. Prochaska JO, DiClemente CC, Norcross JC. In search of how people change. Applications to addictive behaviors. Am Psychol. 1992;47(9):1102-1114. doi:10.1037/10248-026

24. Departament de Salut. Programa per a la prevenció i el control del tabaquisme. Enquesta de consum de tabac, alcohol i drogues, 1998. Enquesta de salut de Catalunya 2002, 2006, 2011, 2012 i 2013. 2013.

25. Sabido M, Sunyer J, Masuet G, Masip J. Hospitalized smokers: Compliance with a nonsmoking policy and its predictors. Prev Med. 2006;43(2):113-116. doi:10.1016/j.ypmed.2006.02.012

26. Nieva G, Gual A, Mondon S, Walther M, Saltó E. Evaluation of brief intervention for smoking cessation in hospitalized patients. Med Clin. 2007;19(128):730-732. doi: $10.1157 / 13106128$

27. Observatorio del sistema de salud de Cataluña. Central de resultados. Ambito hospitalario. Barcelona: Agència de qualitat i avaluació sanitàries de Catalunya. departament 
de salut. Generalitat de Catalunya; 2013.

28. Duffy SA, Scholten RL, Karvonen-Gutierrez CA. The relation of tobacco use during hospitalization to post-discharge smoking cessation among US veterans. Prev Med. 2010;50(5-6):285-287. doi:10.1016/j.ypmed.2010.01.012

29. Zack E. Smoking withdrawal and prolonged hospitalization. Clin J Oncol Nurs. 2002;6(1):7-11. doi:10.1188/02.cjon.7-11

30. Rigotti NA, Clair C, Munafo MR, Stead LF. Interventions for smoking cessation in hospitalised patients. Cochrane Database Syst Rev. 2012;5:CD001837. doi:10.1002/14651858.cd001837.pub2

31.Fiore MC, Jaén CR, Baker TB, ed. Treating tobacco use and dependence: 2008 update. Rockville, MD: US Dept of Health and Human Services; 2008

32. Freund M, Campbell E, Paul C, et al. Increasing smoking cessation care provision in hospitals: A meta-analysis of intervention effect. Nicotine Tob Res. 2009;11(6):650662. doi:10.1093/ntr/ntp056

33. Tong EK, Strouse R, Hall J, Kovac M, Schroeder SA. National survey of U.S. health professionals' smoking prevalence, cessation practices, and beliefs. Nicotine Tob Res. 2010;12(7). doi:10.1093/ntr/ntq071

34. Duaso MJ, Bakhshi S, Mujika A, Purssell E, While AE. Nurses' smoking habits and their professional smoking cessation practices. A systematic review and meta-analysis. Int J Nurs Stud. 2017;67:3-11. doi:10.1016/j.ijnurstu.2016.10.011

35. Martínez C, Castellano Y, Andrés A, et al. Factors associated with implementation of the 5A's smoking cessation model. Tobacco Induced Diseases. 2017;15:41. doi:10.1186/s12971-017-0146-7

36. Kazemzadeh Z, Manzari ZS, Pouresmail Z. Nursing interventions for smoking cessation in hospitalized patients: A systematic review. Int Nurs Rev. 2016;64(2). doi: $10.1111 /$ inr. 12320

37. Campbell S, Pieters K, Mullen KA, Reece R, Reid RD. Examining sustainability in a hospital setting: Case of smoking cessation. Implement Sci. 2011;6. doi:10.1186/1748-5908-6-108

38. Applegate BW, Sheffer CE, Crews KM, Payne TJ, Smith PO. A survey of tobacco-related knowledge, attitudes and behaviours of primary care providers in Mississippi. J Eval Clin Pract. 2008;14(4):537-544. doi:10.1111/j.1365-2753.2007.00910.x

39. Martinez C. Barriers and challenges of implementing tobacco control policies in hospitals: Applying the institutional analysis and development framework to the Catalan network of smoke-free hospitals. Policy Polit Nurs Pract. 2009;10(3):224-232. doi:10.1177/1527154409346736

40. Ballbe M, Martinez C, Salto E, et al. Maintenance of tobacco cessation programmes in public hospitals in Catalonia, Spain. Addict Behav. 2015;42:136-139. doi:10.1016/j.addbeh.2014.11.028

41. Martinez C, Fu M, Martinez-Sanchez JM, et al. Impact of a long-term tobacco-free policy at a comprehensive cancer center: A series of cross-sectional surveys. BMC Public Health. 2014;14. doi:10.1186/1471-2458-14-1228

42. Ballbe M, Mondon S, Nieva G, Walther M, Salto E, Gual A. Evaluation of a training programme for health professionals on smoking cessation in hospitalized. Adicciones. 2008;20(2):125-129. doi:10.20882/adicciones.238

\section{ACKNOWLEDGEMENTS}

The authors would like to thank the participants for contributing to the study, and the participating hospitals and coordinators: The Catalan Institute of Oncology (Tarsila Ferro); Hospital Universitàri Vall d'Hebron (José Maria Sánchez-Garcia, Gemma Nieva); Hospital Sant Joan de Déu d'Althaia (Antònia Raich); Hospital Trias i Pujol (Jorge Sanz); Hospital de Mataró (Margarita Cano); Hospital Clínic i Provincial (Manel Santiñà); Hospital Sant Camil (Joan Prats); Hospital General de Vic (Miquel Vilardell); Hospital del Mar (Consòl Serra); Hospital de Mollet (Gemma Mayor); Hospital de Bellvitge (Josep María Ramon); and Hospital Moisès Broggi (Ruth Ripoll and Àngels Ruz). We also grateful for the support of Francesc Abella and Antònia Raich as external advisors.

\section{CONFLICTS OF INTEREST}

Authors have completed and submitted the ICMJE Form for Disclosure of Potential Conflicts of Interest and none was reported.

\section{FUNDING}

This study was partially funded by the Col-legi Oficial d'Infermeres i Infermers de Barcelona (COIB PR-2619-12). The Tobacco Control Unit was funded by the Government of Catalonia (Directorate of Research and Universities grant 2005SGR00646) and the Government of Spain (Thematic Network of Cooperative Research on Cancer, RTICC grants RD06/0020/0089 and RD12/0036/0053). Two projects lead by C. M. and E. F. have been funded by Global Bridges (GB): Development and Dissemination of a Tobacco Cessation Training Program for Healthcare Professionals in Spanish-speaking Countries (GB-13520139), and Improving smoking cessation interventions in Southern European Countries (GB-25678023). C. M. was also supported by the Instituto de Salud Carlos III, Government of Spain, co-funded by the European Regional Development Fund (FEDER) (INT17/00116) and Ministry of Health from the Government of Catalonia (PERIS No 9015586920/2017). The payment of the Open Access fee was funded by the Col-legi Oficial d'Infermeres i Infermers de Barcelona (ACCO-1462/18).

\section{PROVENANCE AND PEER REVIEW}

Not commissioned; externally peer reviewed. 